\title{
ARTICLE Mitochondrial permeability transition pore induction is linked to formation of the complex of ATPase C-subunit, polyhydroxybutyrate and inorganic polyphosphate
}

\author{
PA Elustondo ${ }^{1}$, M Nichols ${ }^{2}$, A Negoda ${ }^{1}$, A Thirumaran ${ }^{2}$, E Zakharian ${ }^{3}$, GS Robertson ${ }^{2}$ and EV Pavlov ${ }^{1,4}$
}

Mitochondrial permeability transition pore (mPTP) opening allows free movement of ions and small molecules leading to mitochondrial membrane depolarization and ATP depletion that triggers cell death. A multi-protein complex of the mitochondrial ATP synthase has an essential role in MPTP. However, the molecular identity of the central 'pore' part of mPTP complex is not known. A highly purified fraction of mammalian mitochondria containing C-subunit of ATPase (C-subunit), calcium, inorganic polyphosphate (polyP) and polyhydroxybutyrate (PHB) forms ion channels with properties that resemble the native mPTP. We demonstrate here that amount of this channel-forming complex dramatically increases in intact mitochondria during mPTP activation. This increase is inhibited by both Cyclosporine A, an inhibitor of mPTP and Ruthenium Red, an inhibitor of the Mitochondrial Calcium Uniporter. Similar increases in the amount of complex formation occurs in areas of mouse brain damaged by ischemia-reperfusion injury. These findings suggest that calcium-induced mPTP is associated with de novo assembly of a channel comprising C-subunit, polyP and PHB.

Cell Death Discovery (2016) 2, 16070; doi:10.1038/cddiscovery.2016.70; published online 5 December 2016

\section{INTRODUCTION}

The mitochondrial permeability transition pore (mPTP) is a large, weakly selective channel found in the mitochondrial inner membrane. ${ }^{1}$ Opening of the mPTP channel leads to a dramatic increase in the inner membrane permeability, dissipation of the mitochondrial membrane potential and disruption of ATP production that, if not reversed, can trigger cell death. MPTP activation is thought to have a major role in ischemic cell death associated with stroke and myocardial infarction. This hypothesis is supported by the fact that administration of cyclosporine A, an mPTP inhibitor, reduces infarct volume following ischemiareperfusion injury in the heart. ${ }^{2}$ Although these findings suggest that the MPTP is a viable and important drug target, therapeutic efforts in this field have been hampered by a poor understanding of the molecular organization of this channel.

Compelling evidence from several laboratories suggest that the MPTP is associated with, or is an integral part, of a multi-protein complex formed by ATP synthase. ${ }^{3-6}$ More specifically, the C-subunit of ATP synthase, which can form channels in model membranes, ${ }^{6}$ appears to be essential for mPTP opening. ${ }^{5}$ However, the C-subunit alone cannot replicate MPTP activity suggesting that other molecules participate in this complex. The precise nature and molecular organization of the channel part of mPTP remain controversial. ${ }^{7-11}$

We have developed a protocol for the isolation and purification of the mitochondrial channel that closely resembles activity of native mPTP channels. ${ }^{12}$ This protocol, originally described by Reusch and Sadoff, ${ }^{13}$ is a modified solvent extraction for the isolation of the channel formed by inorganic polyphosphate
(polyP), polyhydroxybutyrate (PHB) and calcium in bacteria. In the case of mammalian mitochondria, we have shown that in addition to polyP and $\mathrm{PHB}$, this highly purified fraction also contains the C-subunit. ${ }^{12}$ This channel showed characteristics that resembled the native MPTP with multiple conductance states and a voltage dependence response. Our subsequent studies have confirmed that participation of polyP is essential for the calcium-induced activation of the mPTP. ${ }^{14-17}$

Our central goal was to test the hypothesis that MPTP activation is caused by calcium-induced formation of the complex of C-subunit, polyP and PHB. To achieve this objective, we compared the amounts of the complex purified from control mitochondria and mitochondria with induced MPTP activity. We found that the amount of C-subunit, polyP and PHB complex was increased in mitochondrial preparations with activated MPTP. On the basis of these results, we propose the novel concept that MPTP activation requires de novo calcium-induced assembly of the channelforming complex composed of C-subunit, polyP, PHB and calcium.

\section{RESULTS \\ mPTP induction in isolated mitochondria}

Previous attempts to isolate the MPTP channel have focused on purification of the channel-forming material from isolated mitochondria that are viable and lack MPTP activity. ${ }^{4,6}$ Previously, we proposed the possibility that not only MPTP channel opening but also its formation might be stimulated by mitochondrial calcium uptake, ${ }^{12}$ which was consistent with previous works by Bernardi's group. ${ }^{18}$ If this is the case, components of the channel-

\footnotetext{
${ }^{1}$ Department of Physiology and Biophysics, Faculty of Medicine, Dalhousie University, Halifax, NS, B3H 4R2 Canada; ${ }^{2}$ Departments of Psychiatry and Pharmacology, Brain Repair Centre, Faculty of Medicine Dalhousie University, Halifax, NS, B3H 4R2f Canada; ${ }^{3}$ Department of Cancer Biology and Pharmacology, University of Illinois College of Medicine, 1 Illini Drive, Peoria, IL 61605, USA and ${ }^{4}$ Department of Basic Sciences, New York University, College of Dentistry, 345 East 24 th Street, New York, NY 10010, USA. Correspondence: EV Pavlov (ep37@nyu.edu)

Received 14 June 2016; revised 10 August 2016; accepted 19 August 2016; Edited by R Aqeilan
} 

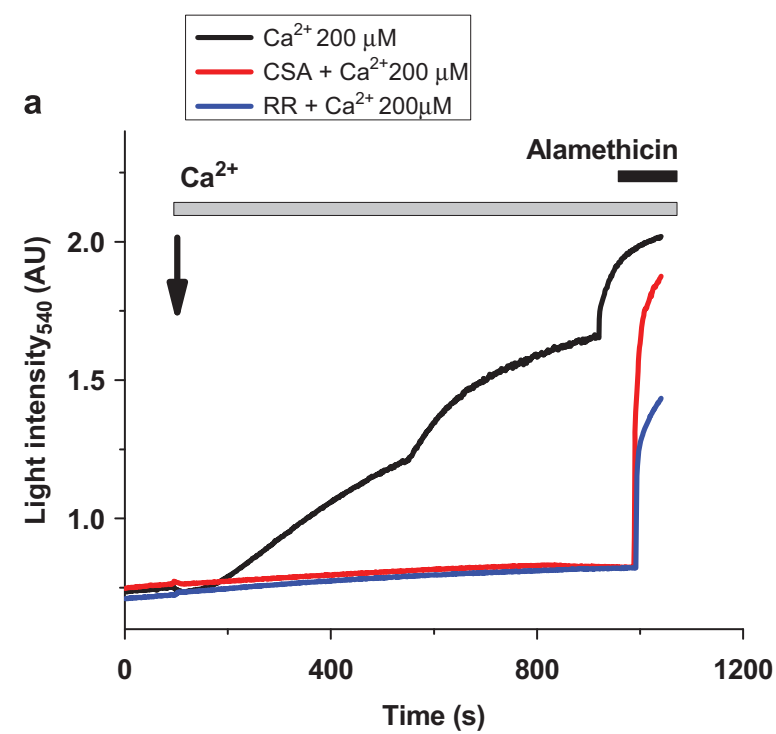

b
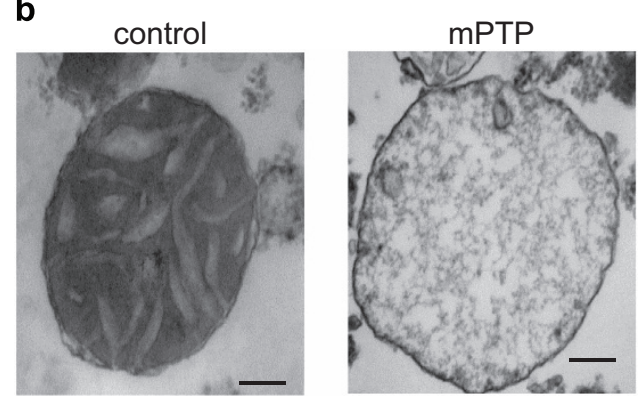

Figure 1. MPTP opening induced by $\mathrm{Ca}^{2+}$ in isolated mitochondria is blocked by CSA and Ruthenium Red. (a) Mitochondrial swelling induced by $\mathrm{Ca}^{2+}$ (detected as an increase in light transmittance) corresponds to MPTP opening (black) as this swelling is blocked by mPTP inhibitor, CSA (red) or by inhibition of the mitochondrial $\mathrm{Ca}^{2+}$ uptake with Ruthenium Red. Alamethicin was added at the end of the experiments to achieve maximal swelling. (b) Electron micrographs of condensed mitochondria before (control) and swollen mitoplasts after mPTP activation (mPTP). Scale bar is $100 \mathrm{~nm}$.

forming fraction should be increased in mitochondria under conditions of calcium-induced mPTP activation. To test this hypothesis, we performed the chemical analysis of the components of the channel-forming complex that demonstrates mPTP activity $^{12}$ in control and mPTP-induced mitochondria. In these experiments, we used isolated rat liver mitochondria. Use of isolated mitochondria rather than cultured cells was advantageous as it allowed us to carefully control $\mathrm{Ca}^{2+}$ load and provided sufficient amount of material for biochemical assays. Before the complex extraction and purification, we induced MPTP in energized isolated mitochondria by the addition of calcium. The MPTP induction was monitored by measuring mitochondrial swelling. Figure 1 shows a representative example of such assays that were routinely performed before biochemical assays described in the following sections. This was required to establish that mitochondria have developed MPTP or that MPTP was inhibited. As can be seen from Figure 1a (black trace), the addition of $200 \mu \mathrm{M}$ of calcium to the mitochondria (1 mg protein $/ \mathrm{ml}$ ) leads to the increase in transmitted light intensity, indicative of mitochondrial swelling. We further confirmed mitochondrial swelling by electron microscopy. Figure $1 \mathrm{~b}$ shows representative electron microscopy images of normal mitochondria with dense matrix and clearly visible cristae ('control') in comparison with the swollen mitochondria with activated mPTP (labeled as 'mPTP'). Note the typical loss of the mitochondrial outer membrane, low electron density and disrupted cristae in the mitochondria of calcium-treated samples (see Elustondo et al. ${ }^{19}$ ). Swelling was not detected in the presence of Cyclosporine A (CSA), an inhibitor of MPTP (Figure 1a, red trace) or Ruthenium Red, an inhibitor of mitochondrial calcium uptake (Figure $1 \mathrm{a}$, blue trace) confirming the involvement of mPTP activation. Alamethicin, a pore forming ionophore, was added at the end of each experiment to confirm mitochondrial integrity. Following mitochondrial treatment and confirmation of MPTP activation, mitochondrial samples were subjected to the dehydration procedure by sequential methanol, methanol/acetone and acetone treatment followed by a chloroform extraction as detailed in the methods section.

Analysis of the components of the channel-forming fraction purified from the calcium-treated mitochondria

As described by our previous work, ${ }^{12}$ the channel-forming fraction of calcium-treated mitochondria contained C-subunit of ATP synthase, polyP and PHB. This fraction showed channel activity when reconstituted in planar lipid bilayer that resembled the native MPTP with multiconductance states and voltage dependence. $^{12}$ Here we compared the levels of all these compounds in purified control and mPTP-activated mitochondria. To make sure that equal amounts of material were used, equal volumes of the mitochondrial sample originally taken from the same tube of the isolated mitochondria were used in all the experiments presented in the figures. This allowed us to exclude any variability that might have occurred due to different amounts of mitochondria or their different functional state.

Quantification of C-subunit in the channel-forming fraction of the extract was analyzed using immunoblotting assays with C-subunit antibodies. As can be seen from Figures $2 a$ and $b$, the extracts from mitochondria with activated MPTP contained significantly higher amounts of C-subunit compared with control mitochondria $(n=3, P<0.01)$. Importantly, when mitochondria were treated with the same amount of calcium in the presence of the MPTP inhibitor Cyclosporine A, C-subunit levels were not increased compared with the C-subunit levels detected in control and Cyclosporine A-treated mitochondria. Similarly, C-subunit levels were not increased when calcium treatment was done in the presence of Ruthenium Red - an inhibitor of mitochondrial calcium uptake. These results support the idea that the transfer of C-subunit into the chloroform extract is directly related to mitochondrial calcium uptake and activation of the MPTP. Taking into account that with the exception of $\mathrm{C}$-subunit this extraction protocol leads to the sample with non-detectable levels of protein, ${ }^{12}$ it was not possible to use endogenous protein as a loading control. To make sure the samples were comparable, the total amount of extract, which in each experiment is originated from the same amount of mitochondria, was resuspended in equal volumes and run on the same gel. To confirm that the increase in the detection of $C$-subunit was not a consequence of a change in the amount of total protein occurrence as a consequence of the $\mathrm{Ca}^{2+}$ treatment, we studied the amount of C-subunit directly immunoprecipitated from the whole mitochondrial lysates exposed to high or no $\mathrm{Ca}^{2+}$. As can be seen from the immunoblot results, the total amount of $C$-subunit did not change after calcium treatment (Figure 2c). These control experiments demonstrate that there is no change in the total C-subunit levels present in the sample regardless of the treatment condition. We were also concerned that the recovery of higher amounts of C-subunit could be a consequence of a difference in the membrane integrity caused by the mitochondrial swelling of the preparation so we included a control where we lyzed the mitochondria with a buffer containing detergent $(500 \mathrm{mM} \mathrm{NaCl}$, $50 \mathrm{mM} \mathrm{NaH} \mathrm{PO}_{4}, 20 \mathrm{mM}$ Hepes and 10\% glycerol, pH 7.4). We did 
a
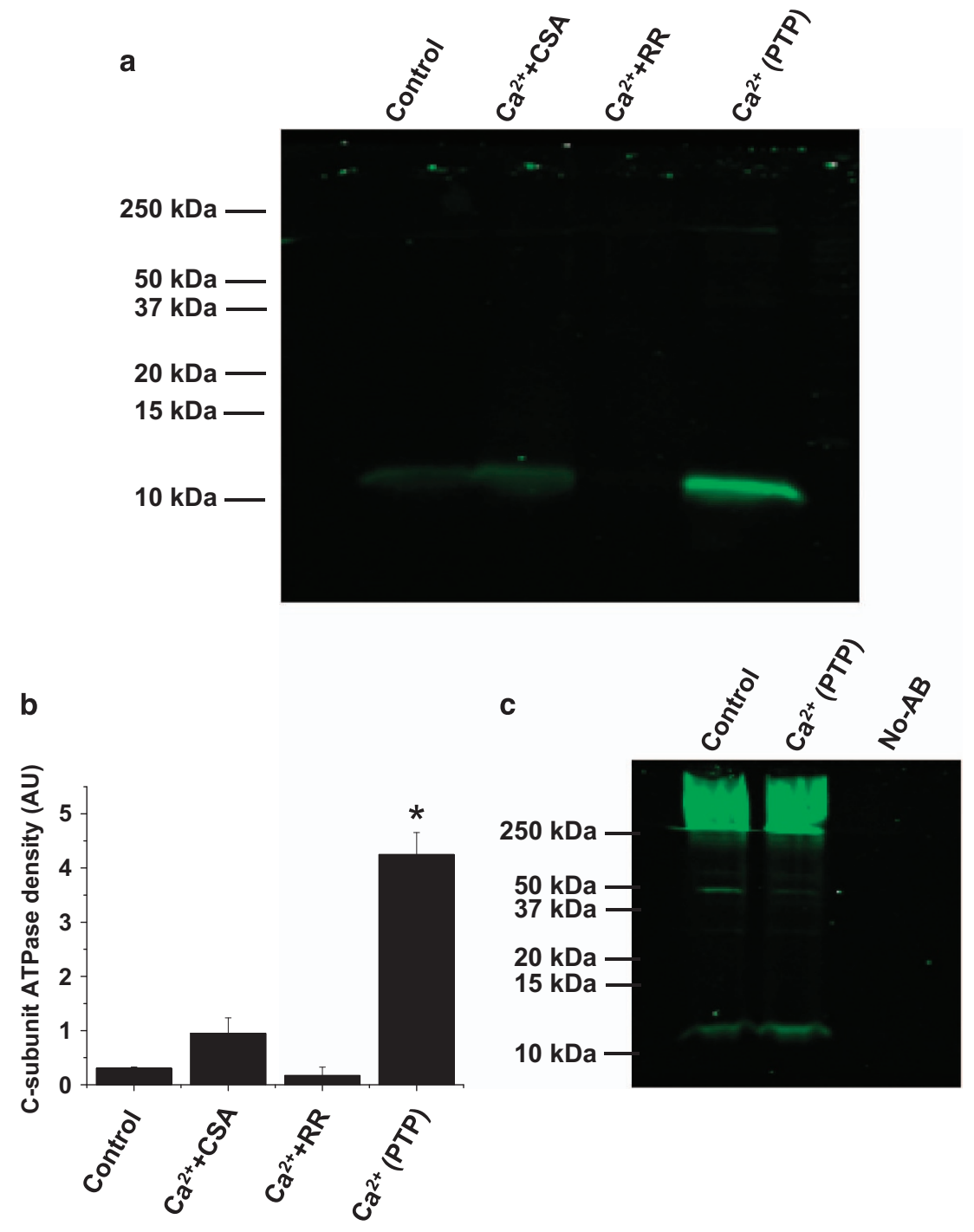

Figure 2. mPTP activation increases C-subunit content of the channel-forming fraction (chloroform extractable C-subunit). (a) Western blot using C-subunit-specific antibodies shows that MPTP activation in isolated mitochondria $\left(+\mathrm{Ca}^{2+}\right)$ increases the amount of C-subunit associated with channel-forming 'PTP pore' fraction. Note that the amount of complex is dramatically decreased if mPTP is inhibited with $5 \mu$ M Ruthenium Red (Rut Red) or $1 \mu \mathrm{M}$ CSA despite the presence of the same amounts of $\mathrm{Ca}^{2+}(200 \mu \mathrm{M})$. The samples for analysis were purified using chloroform extraction protocol as described in Pavlov et al. ${ }^{12}$ and identical to the samples used for mPTP monitoring in Figure 1. (b) Densitometry analysis of western blots (as in a) from three independent experiments $(* P<0.01$ t-test). (c) Western blot of C-subunit immunoprecipitated from the total mitochondrial lysate using C-subunit antibodies shows similar total amounts of C-subunit in different samples. Note high molecular bands presumably formed by oligomers of the C-subunit.

not detect an increase in C-subunit in the chloroform extract from this preparation (not shown). The increase in the relative amount of C-subunit detected in induced mPTP fractions, but not in control fractions, supports a role for the C-subunit in the MPTP channel-forming fraction of mitochondria.

Next, we analyzed the amount of PHB in the chloroform extracts. In contrast to polyP, PHB is not soluble in water and can only be dissolved in nonpolar organic solvents. First, we used thin- layer chromatography (TLC) to separate the nonpolar content of the chloroform extract. Figure 3 a shows the relative distribution of synthetic phospholipids and synthetic PHB standard on the TLC plate. Due to its higher hydrophobicity, PHB migrates significantly faster compared with phospholipids. As can be seen from Figure $3 b$, the chloroform extract contained only the material that migrated at the same rate as the PHB standard. In both Figures $3 a$ and $b$, this material was visualized by nonspecific iodine 
a

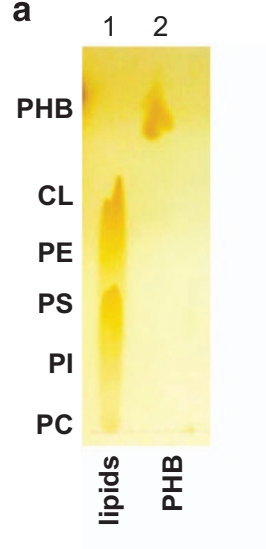

b 12

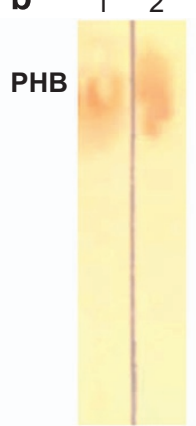

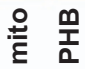
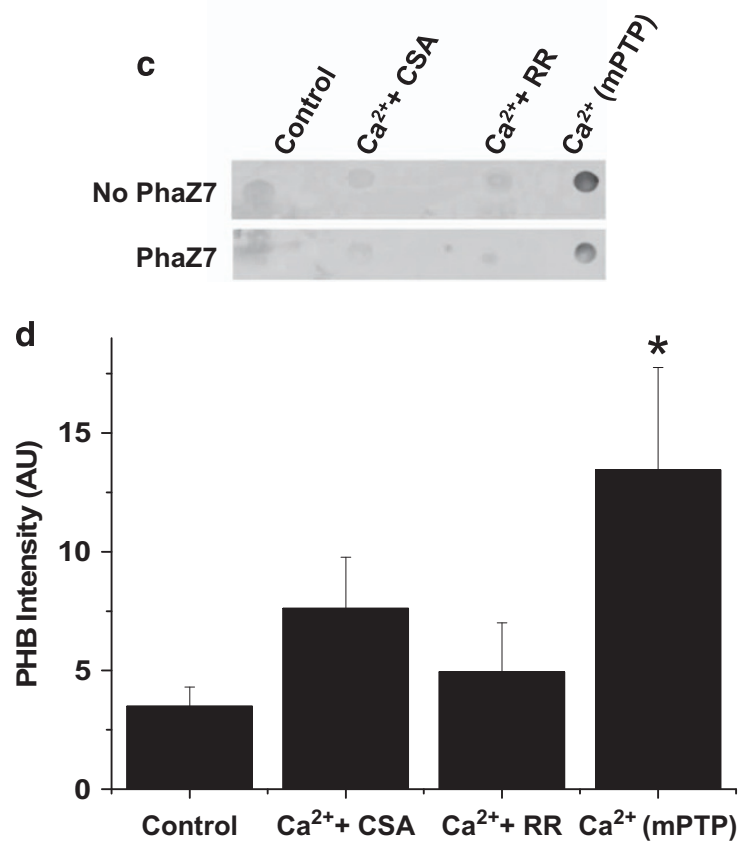

Figure 3. $\mathrm{PHB}$ is present in channel-forming fraction and is increased upon activation of mPTP. (a) Thin-layer chromatography (TLC) showing a mix of phospholipid standards (lane 1, CL: cardiolipin; PE phosphatidylethanoamine; PI, phosphatidylinositol; PS, phosphatidylserine) and synthetic PHB standard (lane 2). (b) TLC showing mitochondrial extract (lane 1) and a PHB standard (lane 2). Both TLCs were stained with lodine. (c) PHB detection using dot blot immunostaining of the extracts using PHB antibodies. Note that the signal was reduced by PHB-depolymerase (PhaZ7) treatment. PHB was present in higher amounts in calcium-treated mitochondria $-\mathrm{Ca}^{2+}$ (mPTP)- compared with control or mitochondria pretreated with Cyclosporin A (+CSA) or Ruthenium Red (+RR). (d) Densitometry analysis of dot blots from four independent experiments showing a significant increase in the recovery of PHB in the extract from mitochondria treated with calcium when compared with control, RR- or CSA-treated mitochondria ( $n=4,{ }^{*} P<0.05, t$-test).

staining. On the basis of the TLC, the retention factors (Rf) of the hydrophobic material in the channel-forming fraction (Rf 0.6-0.9) were similar to the PHB standard ( $\operatorname{Rf} 0.6-0.8$ ). To confirm the presence of PHB, we blotted the sample onto a PVDF membrane and visualized this molecule using PHB-specific antibodies. Figures $3 c$ and $d$ show that the channel-forming fraction from MPTP-activated mitochondria contained significantly higher amounts of PHB compared with samples isolated from control mitochondria or from mitochondria in which the MPTP was inhibited with either CSA or ruthenium red treatment. Signal

intensity was decreased by treatment of the sample with a specific PHB-hydrolyzing enzyme (PHB depolymerase, PhaZ7 ${ }^{(\text {ref.20) }}$ ) further confirming that the detected signal reflects the presence of $\mathrm{PHB}$ (Figure 3c). Finally, we measured the amounts of polyP in the chloroform extracts of control and calcium-treated mitochondria. To detect polyP, the extract was transferred from a chloroform soluble ('complex' form) phase into a water soluble ('free' form) phase. This was achieved by the incubation of the chloroform phase with water. This procedure allows dissociation of the PHB/ polyP complex, transfer of the polyP into the polar water phase and retention of PHB in the chloroform phase. PolyP was detected in the water phase with a DAPI-based fluorescence assay. When polyP binds to DAPI, it induces a shift in the excitation and emission peaks characteristic for polyP. ${ }^{21}$ As can be seen from Figures $4 a$ and $b$, the amount of poly $\mathrm{P}$ was significantly higher in the samples from calcium-treated mitochondria compared with control mitochondria. The fluorescent spectrum of the sample was similar to the spectrum for synthetic polyP (Figure 4c). On the basis of the calibration curve performed using polyP standards, we estimate that the amount of polyP extracted from $1 \mathrm{~g}$ of liver is approximately $1 \mu \mathrm{g}$. The fluorescent signal was eliminated from the sample by treatment with alkaline phosphatase to induce polyP hydrolysis (Figure $4 \mathrm{~d}$ ) thus confirming that it reflects the amount of polyP present in the sample. However, the increase in polyP was only observed when the process of dehydration was done in the presence of high amounts of calcium $(10 \mathrm{mM})$. We hypothesize that polyP is required in the process of pore formation but is easily dissociated from the complex as soon as the local concentration of calcium decreases following mPTP activation and its efflux from the matrix. PolyP is a highly charged polyanion with excellent water solubility making extraction with chloroform only possible if this polymer is complexed with hydrophobic molecules. We have previously shown that the ability of polyP to be extracted with chloroform from mPTP-activated mitochondria is determined by the presence of $\mathrm{PHB} .^{12}$ Altogether, these findings indicate that the induction of MPTP activity in mitochondria is accompanied by increased levels of PHB, polyP and C-subunit known to have channel-forming activities.

Direct extraction of C-subunit by chloroform from Hypoxic-Ischemic-injured brain

The use of isolated mitochondria allowed us to establish the direct link between $\mathrm{Ca}^{2+}$ mPTP and formation of the PHB, polyP and C-subunit complex. The activation of the MPTP is thought to have a pivotal role in the pathophysiology of ischemia-reperfusion injuries. For this reason, we utilized an ischemia-reperfusion model of stroke in mice to investigate the contributions of polyp, PHB and c-subunit in vivo. The activation of MPTP is considered to have a central role in ischemic cell death responsible for brain damage after a stroke (reviewed in Bernardi et $a l^{22}$ ). We investigated whether increased mPTP channel formation, observed at the level of isolated mitochondria, is also induced by $\mathrm{HI}$ brain damage in mice. Before the use of this animal model, we tested the feasibility of the extraction method by analyzing the channel-forming fraction purified directly from the whole liver without prior mitochondrial isolation. The amount of C-subunit in chloroform extracts were examined in whole livers homogenized in the presence and absence of EGTA. In the absence of EGTA during homogenization, mitochondria are exposed to high levels of calcium that induce opening of mPTP. Analysis of the extracts by dot blot using C-subunit antibodies (Supplementary Figure 1) showed that in the presence of high calcium, C-subunit levels in extracts from whole liver were significantly higher compared with those observed in control extracts isolated from tissues homogenized in the presence of EGTA. Consistently higher amounts of $\mathrm{PHB}$ were recovered in the extracts from calcium-exposed livers. The presence of PHB was confirmed by the decrease in signal 
a

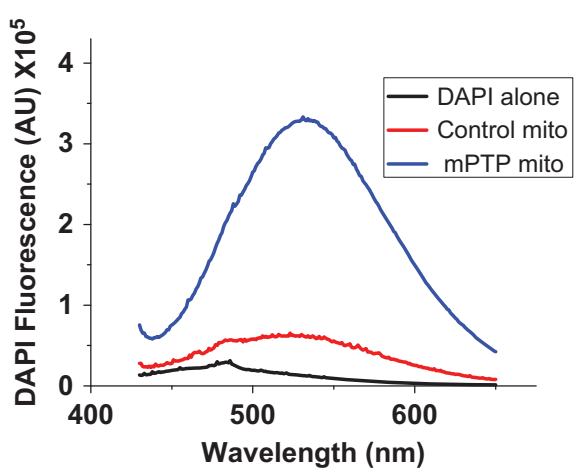

C

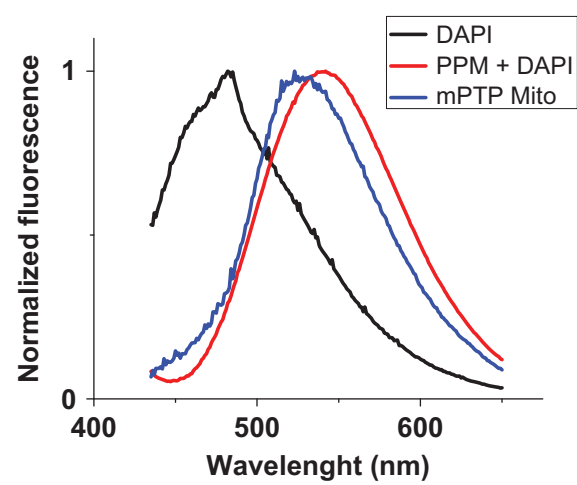

b

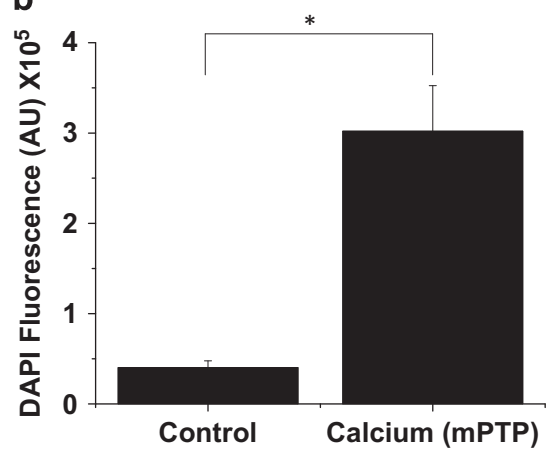

d

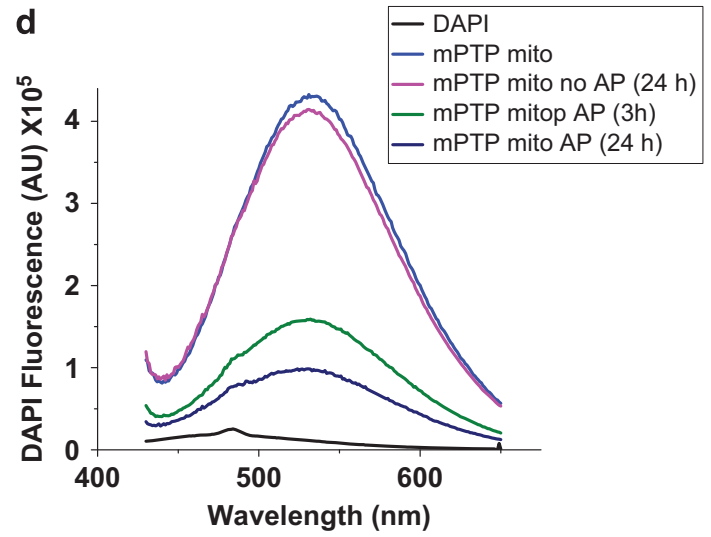

Figure 4. mPTP activation increases polyP levels in the channel-forming fraction. (a) Emission spectra of DAPI in the presence of the extracts purified from mitochondria treated with $10 \mathrm{mM} \mathrm{Ca}^{2+}$ to induce mPTP (blue) and control (red). The black line shows the emission spectrum of DAPI alone. (b) Combined data of the values of peak DAPI fluorescence from three independent experiments. Note the significant increase in the amount of polyP in treated calcium samples (blue) when compared with control (red; $t$-test, $\left.{ }^{*} P<0.002\right)$. (c) Comparison of the emission spectra of DAPI alone (black), synthetic polyP (PPM, 60 phosphates, red) and purified fraction (blue); (d) Alkaline phosphatase (AP) treatment shows a decrease in the DAPI fluorescence confirming the presence of polyP.

when the samples were treated with a specific PHB depolymerase, PhaZ7 (Supplementary Figure 1B).

On the basis of these results, we hypothesized that the amount of $\mathrm{C}$-subunit recovered in a water-free chloroform extract from the $\mathrm{HI}$-injured hemisphere would be greater relative to that obtained from the non-injured hemisphere. To test this hypothesis, mice were subjected to unilateral common carotid artery occlusion followed by exposure to a low oxygen ( $\left.8 \% \mathrm{O}_{2}, 50 \mathrm{~min}\right)$ environment to induce $\mathrm{HI}$ brain injury. Fluoro-Jade (FJ) staining, which positively labels degenerating neurons, was performed to quantify the level of neuronal damage $24 \mathrm{~h}$ following $\mathrm{HI}$. A representative tiled image at the level of the midstraitum is shown to demonstrate the size of the (Figure $5 \mathrm{a}$ ). Figure $5 \mathrm{~b}$ shows FJ positive staining in the damaged striatum. Mitochondrial injury was also assessed by direct visualization of mitochondria by electron microscopy. EM images of control hemisphere (Figures $5 \mathrm{c}$ and e) display mitochondria with well-formed cristae and doublemembrane structures. In contrast, the mitochondria from the infarcted hemisphere display a loss of cristae and membrane integrity (Figures $5 \mathrm{~d}$ and $\mathrm{f}$ ). TTC staining indicated that the infarct produced by $\mathrm{HI}$ occupied about $50-70 \%$ of the affected hemisphere (Figure 6a). The contralateral (non-injured) and ipsilateral (injured) brain hemispheres from mice subjected to unilateral $\mathrm{HI}$ injury were collected in methanol and extracted using the waterfree chloroform extraction method. The extracts were analyzed by dot blot with anti-C-subunit and anti-PHB antibodies. Figure $6 \mathrm{~b}$ is a representative image from an HI-injured mouse showing that relative to an extract from the uninjured (control) hemisphere, the amount of C-subunit appears to be elevated in the injured hemisphere. This finding was confirmed by the quantification of $\mathrm{C}$-subunit levels in the control and injured hemispheres of $16 \mathrm{HI}$ mice (Figures 6c, $P<0.05$, Mann-Whitney nonparametric test). Interestingly, there was no significant difference in the amount of $\mathrm{PHB}$ recovered in these samples when the injured and non-injured hemispheres were compared (Supplementary Figure 1C). This result may reflect the high abundance of $\mathrm{PHB}$ in brain.

\section{DISCUSSION}

Recent models of mPTP generally agree that ATP synthase has an essential role in formation of the MPTP. ${ }^{3,8,9}$ Moreover, genetic manipulations of the C-subunit of ATP synthase correlate with alterations in MPTP activity. ${ }^{6}$ This suggests that the C-subunit of the ATP synthase is an essential component of the MPTP channel. The C-subunit is an oligomer that forms a barrel-like structure in the lipid bilayer that could comprise the mPTP pore. Consistent with this proposal, purified C-subunit can form ion-conducting pores albeit their characteristics do not closely resemble native mPTP activity. ${ }^{23}$ We showed, in previous work, that PolyP is also required for MPTP opening in primary neuronal cultures and cardiac myocytes. ${ }^{16,17}$ Furthermore, when added to cultured cells, PHB is targeted to the mitochondria where it induces mPTP. ${ }^{24}$ Our demonstration that comparable elevations of C-subunit of ATPase, polyP and PHB were detected in chloroform extracts from MPTP- 

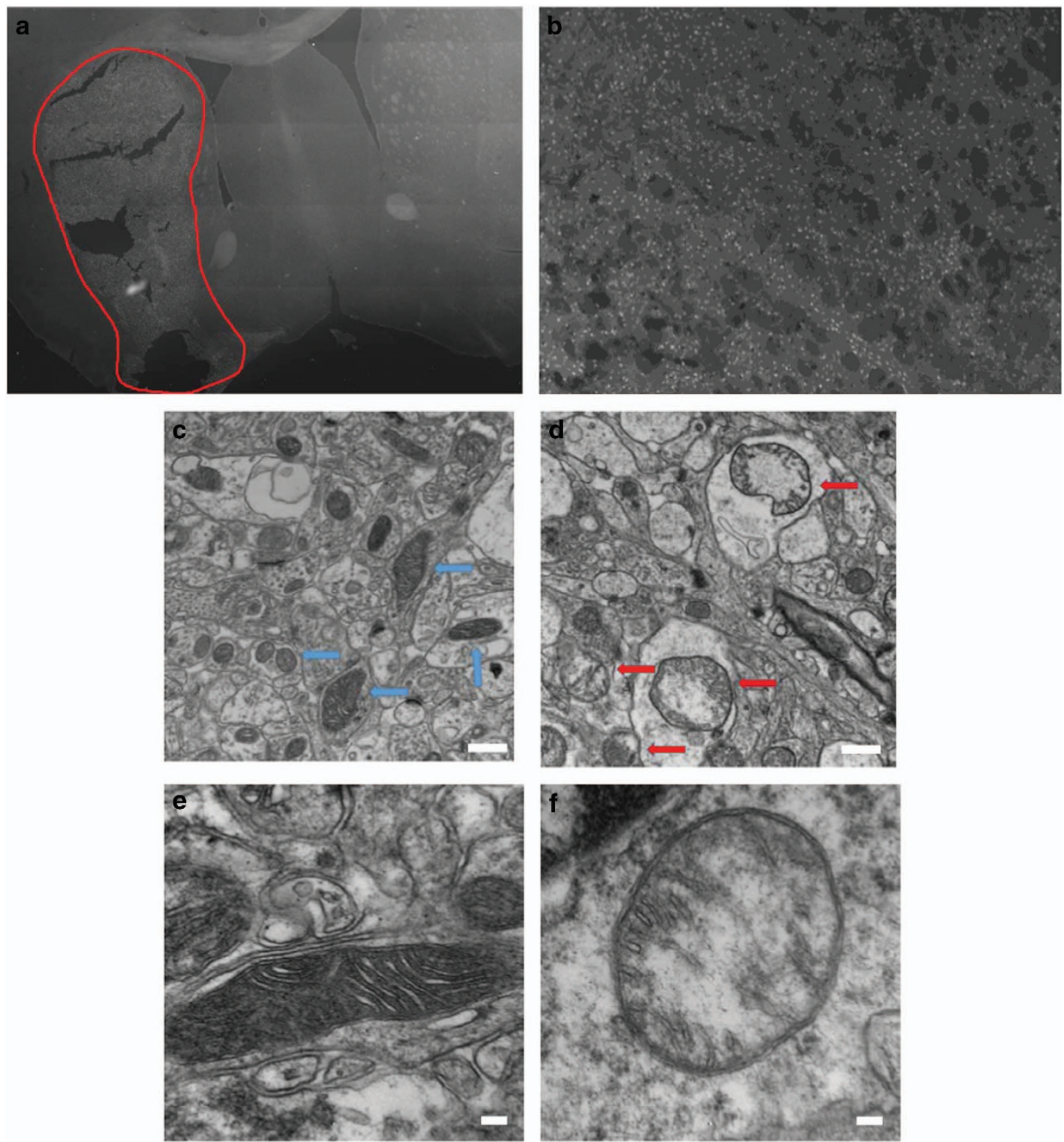

Figure 5. Characterization of the cell and mitochondrial damage following ischemia. (a) Representative $10 \times 10$ tile stained with Fluoro-Jade to identify degenerating neurons. The picture is representative of the damage observed in the striatum of animals following HI-induced brain injury. The damaged area is outlined by the red line. (b) Image of an individual tile at $\times 10$ magnification representative of the damage observed in the striatum. (c) EM image of control brain tissue with fully intact mitochondria marked with blue arrows. (d) EM image of damaged area showing damaged mitochondria with loss of normal cristae structure, marked by red arrows. (e and f) EM images of control and damaged mitochondria at increased magnification. Scale bars are $500 \mathrm{~nm}$ for $\mathbf{c}$ and $\mathbf{d} ; 100 \mathrm{~nm}$ for $\mathbf{e}$ and $\mathbf{f}$.

activated mitochondria supports an important role of these polymers in MPTP activity. By contrast, when $\mathrm{Ca}^{2+}$-induced mPTP activation was blocked with the $\mathrm{Ca}^{2+}$ uptake inhibitor Ruthenium Red, C-subunit was significantly lower in the extract. The reliance of channel formation on mitochondrial calcium uptake suggests that $\mathrm{Ca}^{2+}$ is exerting its effect in the mitochondrial matrix space. Inhibition of the $\mathrm{Ca}^{2+}$-induced mPTP activation with Cyclosporine $A$ also reduced $C$-subunit levels in the chloroform extract. The in vivo relevance of these findings is supported by the elevation of C-subunit levels in chloroform extracts from the damaged hemisphere after an experimental stroke. In this animal surgical model, the stroke was induced by restricting the blood circulation to one hemisphere of the brain, followed by $50 \mathrm{~min}$ of hypoxia. This protocol induces the opening of $\mathrm{mPTP} .{ }^{25}$ On the basis of these results, we propose that formation of the $\mathrm{MPTP}$ requires a calcium-induced assembly of the complex composed of C-subunit, polyP and PHB. These findings suggest that induction of the MPTP is linked to a change in $\mathrm{C}$-subunit association with these polymers rather than a change in the total amount of the C-subunit or in its conformation. This interpretation likely explains why the C-subunit is not found in the purified fraction of the control mitochondria. Indeed, when C-subunit is not complexed with PHB and polyP, this protein likely dissolves in the acetone phase during the dehydration step and is therefore not present in the pellet used for channel extraction by chloroform. In contrast, when the C-subunit associates with polyP and $\mathrm{PHB}$, this protein is rendered soluble in the chloroform phase.

The essential requirement of interaction between three components ( $\mathrm{C}$-subunit, polyP and $\mathrm{PHB}$ ) to form the functional 
a

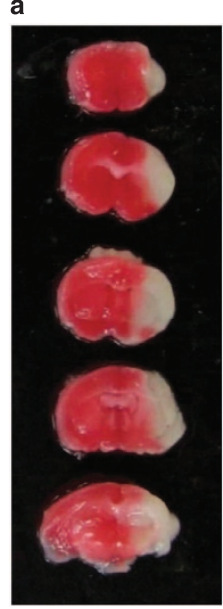

b
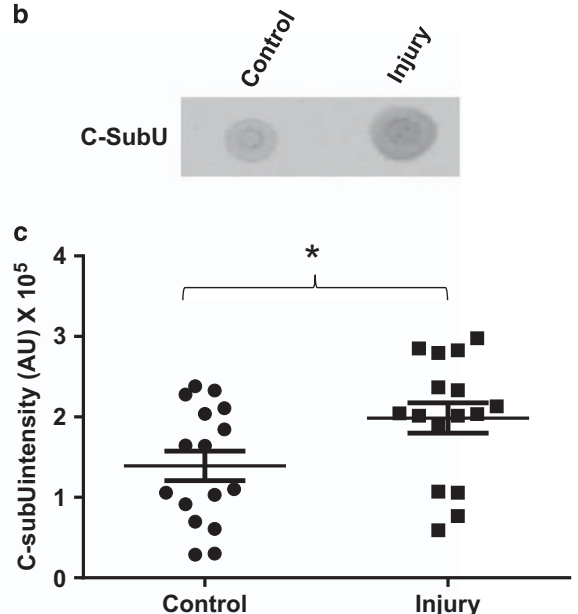

Figure 6. HI brain injury is associated with increased C-subunit levels in the channel-forming fraction. (a) Image of a whole brain stained with TTC (2,3,5-triphenyltetrazolium chloride) from a representative mouse subjected to $\mathrm{HI}$ brain injury. Viable tissue is stained red while the infarct is unstained or white. (b) Dot blot for C-subunit prepared using C-subunit antibodies. The extracts from the injured side of the brain showed higher amounts of C-subunit compared with the noninjured side. (c) Densitometry analysis of the C-subunit dot blots of chloroform extracts from brain uninjured (control) and damaged (injury) hemispheres showing increased C-subunit levels in the damaged hemisphere $\left({ }^{*} P<0.05\right.$, Mann-Whitney nonparametric test, $n=16$ animals).

mPTP provides a mechanism to resolve several controversial points:

1. C-subunit can form large pores of eight C-subunits in mammalian cells. ${ }^{26}$ However, the C-subunit is a highly hydrophobic protein that is not expected to be able to form water-filled pores. This suggests that, on its own, the C-subunit probably cannot assemble into the functional mPTP. Furthermore, unlike native MPTP, the C-subunit alone is not expected to have significant voltage dependence owing to the lack of charged groups necessary to gate ion conductance. The ability of polyP to generate channels with significant voltage dependence and selectivity ${ }^{27-29}$ suggests that this charged polymer may serve as the hydrophilic lining of the MPTP pore largely responsible for its key electrophysiological properties. Furthermore, our previous studies have shown that the same channel demonstrates a transition from a large pore resembling C-subunit channel into a small channel resembling activity of the synthetic polyP/PHB channel in a voltagedependent manner. ${ }^{12}$ The molecular function of channels that involve PHB, polyP and calcium have been described in details and confirmed experimentally by Reusch and Seebach. ${ }^{28,30} \mathrm{We}$ hypothesize that similar principles govern the function of the mitochondrial mPTP channel, but in this case, the poly $/ \mathrm{Ca}^{2}$ ${ }^{+} / \mathrm{PHB}$ channel is likely formed association with the C-subunit ring.

2. It is known that activation of MPTP is caused by an excessive accumulation of calcium. Despite this calcium accumulation, mPTP can be activated while concentrations of free calcium remain constant due to the formation of calcium phosphate complexes. ${ }^{31}$ A recent study indicates that polyP might have an important role in the regulation of this process. ${ }^{32}$ Thus, at the level of intact mitochondria, MPTP is not a channel directly activated by a simple increase in bioavailable calcium but rather by calcium phosphate species. Taking into account that polyP is a 'condensed' form of phosphates and that there is an increase in the formation of calcium phosphate complexes before MPTP opening, it is tempting to speculate that calcium polyP, rather than calcium phosphate interactions, are the direct cause of MPTP formation and opening. More detailed discussion of this model in comparison with other current views can be found in Solesio et al. ${ }^{33}$

3. Finally, patch-clamp studies indicate that very few mPTP channels are present in the mitochondria. ${ }^{34,35}$ However, mitochondria contain high amounts of C-subunit oligomers, which is inconsistent with the relatively rare detection of MPTP. As can be seen from Figure 2, only a small amount of the C-subunit is associated with polyP in the fraction capable of forming MPTP-like channels. We hypothesize that only a small amount of C-subunit participates in complex formation with polyP and PHB. This proposal provides an explanation for the discrepancy between the large amount of C-subunit and the small amount of mPTP channels per mitochondrion.

In conclusion, our results suggest that the assembly of a complex composed of the C-subunit, polyP and PHB is required for mPTP opening which is induced by calcium in the case of isolated mitochondria or by $\mathrm{HI}$ brain damage, presumably also by a calcium overload mechanism. We propose that activation of the mPTP is linked to the formation of the de novo complex, which is not normally present in functional mitochondria. Elucidation of the components of the MPTP provides a model that will assist the development of therapeutics for the prevention of ischemic brain or heart injury.

\section{MATERIALS AND METHODS}

\section{Animals}

Sprague Dawley rats and C57bl/6 mice were purchased from Charles River (Montreal, Quebec, Canada) and housed in a climate-controlled environment with appropriate light-dark cycles. All experimental animals were allowed to eat standard chow and drink water ad libitum. All the procedures were approved by the Animal Care Committee of Dalhousie University in accordance with standards of the Canadian Council on Animal Care. Isolated mitochondria were obtained from rats (6-10 weeks of age) killed with isoflurane/ $\mathrm{CO}_{2}$.

\section{Antibodies}

The following antibodies were used: anti APTase Subunit C (ab181243, Abcam, Cambridge, MA, USA); anti-PHB (gift from Dr Reusch, Michigan State University); Alexa Fluor_ 680 goat antimouse IgG (catalog number A21057, Invitrogen, Carlsbad, CA, USA); Alexa Fluor 750 goat anti-rabbit IgG (catalog number A21039, Invitrogen) and anti-ATPase immunocapture antibody (ab1099867, Abcam, Cambridge, UK).

\section{Isolation of mitochondria}

Mitochondria were isolated from the liver of Sprague Dawley rats by differential centrifugation as previously described. ${ }^{19}$ Briefly, liver from rats was homogenized using Teflon-glass homogenizer and resuspended in $50 \mathrm{ml}$ of isolation buffer (300 mM Sucrose, $5 \mathrm{mM}$ Hepes- $\mathrm{KOH}, 5 \mathrm{mg} / \mathrm{ml} \mathrm{BSA}$, $0.2 \mathrm{mM} \mathrm{KH}_{2} \mathrm{PO}_{4}, 1 \mathrm{mM}$ EDTA, pH 7.4). An initial centrifugation at $600 \mathrm{~g}$ for $15 \mathrm{~min}$ was followed by centrifugation of the supernatant at $6500 \times \mathrm{g}$ for 20 min at $4{ }^{\circ} \mathrm{C}$. The resulting pellet was washed with buffer without EDTA and resuspended in $1 \mathrm{ml}$ of washing buffer $(300 \mathrm{mM}$ sucrose, $5 \mathrm{mM}$ Tris$\mathrm{ClH}, 7.4)$.

mPTP complex induction and extraction from mitochondria Isolated mitochondria were resuspended in recording buffer $(70 \mathrm{mM}$

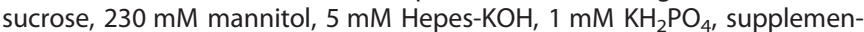
ted with $0.8 \mu \mathrm{M}$ Rotenone, $5 \mathrm{mM}$ Succinate, $\mathrm{pH}$ 7.4). The concentration of calcium needed to be added to the mitochondrial suspension for MPTP activation was determined, after each isolation, by monitoring swelling and was generally in the range of $100-200 \mu \mathrm{M}$. Chloroform extraction of the complex was performed as previously described. ${ }^{12}$ Briefly, isolated mitochondria were resuspended in methanol, incubated for $10 \mathrm{~min}$ on 
ice, and then spun down at $4700 \times g$ for $20 \mathrm{~min}$ (twice). The procedure was repeated with methanol/acetone, 1:1 (twice), and then with acetone (twice). Traces of acetone were removed from the final pellet with a stream of dry nitrogen. Typically, chloroform extraction was done by incubating the dried pellet in $5 \mathrm{ml}$ chloroform at $4{ }^{\circ} \mathrm{C}$ for $48-72 \mathrm{~h}$ in Teflon tubes (Nalgene, Rochester, NY, USA). All solvents used during the process of dehydration and extraction were ice cold, and were pretreated with molecular sieve beads, 8-12 mesh (No. 208604, Sigma-Aldrich, St Louis, $\mathrm{MO}, \mathrm{USA}$ ) to eliminate all traces of water. All the procedures were performed on ice. Before the extracted fraction was resuspended in sample buffer, the chloroform suspension was filtered with a $0.2 \mathrm{~mm}$ PTFE filter (Acrodisc Syringe Filter, PALL Life Sciences, East Hills, NY, USA) using a Teflon syringe. The filtered extract was dried under a nitrogen stream and resuspended in the appropriate buffer for the experiment.

\section{Protein assay}

Isolated mitochondria were resuspended in $100 \mu \mathrm{l}$ of lysis buffer consisting of (in mM): 50 Tris, $150 \mathrm{NaCl}, 50 \mathrm{Na}_{2} \mathrm{HPO}_{4}, 1 \mathrm{Na}_{3} \mathrm{VO}_{4}, 1 \mathrm{NaF}$ and $0.1 \%$ Nonidet P-40, and $0.25 \%$ sodium deoxycholate. The lysis buffer also contained $1 \mu \mathrm{l}$ of a protease inhibitor mixture (Sigma-Aldrich, catalog number P8340). The samples were homogenized and incubated at $4{ }^{\circ} \mathrm{C}$ with constant agitation for $30 \mathrm{~min}$, then centrifuged at $12000 \times \mathrm{g}$ for $15 \mathrm{~min}$. Protein concentration was determined in the supernatant of each sample with a modified Lowry method (Bio-Rad, Hercules, CA, USA; DC Protein Assay, catalog number 500-0116).

\section{Thin-layer chromatography}

TLC was performed on silica gel G (Analtech, Newark, DE, USA). Mitochondrial extracts were resuspended in $50 \mu \mathrm{l}$ chloroform and $10 \mu \mathrm{l}$ aliquots were applied to the TLC plates. The plates were analyzed using chloroform: methanol:water (90:10:0.5) as the solvent system. The staining was performed with iodine vapors.

\section{Immunoblot analysis}

Following protein quantification (DC Protein Assay, Bio-Rad, catalog number $500-0116), 200 \mu \mathrm{g}$ of protein was boiled with loading buffer with $5 \% \beta$ Mercaptoethanol and separated with12\% SDS-PAGE gels and transferred to polyvinylidene fluoride membranes (catalog number IPFL00010 Immobilon, FL, Millipore, Billerica, MA, USA). In the case of the extracts, the samples were resuspended in $25 \mu \mathrm{l}$ of $100 \mathrm{mM}$ Tris buffer, $0.05 \%$ SDS, pH 7.4 The membranes were stained with Ponceau red to assess loading; after they were rinsed with Tris-buffered saline/Tween 20 (TBST; $20 \mathrm{mM}$ Tris base, $137 \mathrm{mM} \mathrm{NaCl}$ and $0.1 \%$ Tween 20, pH 7.6), the blots were blocked in blocking buffer (Oddissey catalog number 927-40000) for $1 \mathrm{~h}$. The blots were incubated with antibodies for ATP synthase subunit $C$ (catalog number ab47010, Abcam), overnight at $4{ }^{\circ} \mathrm{C}$ with constant agitation. Following three washes with PBST, the immunoblots were incubated with either Alexa Fluor 680 goat antimouse IgG (catalog number A21057, Invitrogen) or Alexa Fluor 750 goat anti-rabbit lgG (catalog number A21039, Invitrogen) depending on the primary antibody used. The bands were detected with Odyssey version 3.0, and multiple exposures were generated to ensure the linearity of the fluorescent signal.

Immunoprecipitation was performed with Pierce (Waltham, MA, USA) IgA magnetic beads (catalog number 88846) according to a standard protocol. ${ }^{36}$ Briefly, $500 \mu \mathrm{g}$ of proteins from the whole mitochondrial lysate were incubated overnight with the anti-ATP synthase lgG conjugated to protein A magnetic beads (catalog number 88846, Pierce, Thermo Scientific. The samples were then boiled in SDS-loading buffer and equal amounts of sample were loaded in each lane including a no-antibody control. Immunoblot analysis was done with the anti-subunit-C antibody.

\section{Transmission electron microscopy}

Two hours following $\mathrm{HI}$, the mice were humanely killed with an overdose of pentobarbital $(150 \mathrm{mg} / \mathrm{kg})$. The brains were perfused with PBS followed by fixation in $2.5 \%$ glutaraldehyde in PBS ( $\mathrm{pH} 7.4$ ) and processed by the EM Core Facility (Dalhousie University). In short, the samples were rinsed $3 \times$ in $0.1 \mathrm{M}$ sodium cacodylate buffer and fixed in $1 \%$ osmium tetroxide for $2 \mathrm{~h}$, then dehydrated and embedded in epon araldite resin. Hundred nanometer sections were cut with an ultramicrotome and placed on 300 -mesh copper grids. The sections were stained with $2 \%$ aqueous uranyl acetate, rinsed and treated with lead citrate, rinsed again and air dried. The images were captured with a Jeol Jem 1230 transmission electron microscope at $80 \mathrm{kV}$ attached to a Hamamatsu (Middlesex, NJ, USA) ORCA-HR digital camera.

\section{FJ staining}

The mice were injected with a lethal overdose of sodium pentobarbital $(150 \mathrm{mg} / \mathrm{kg}$, i.p.) and perfused transcardially with PBS $(5 \mathrm{ml})$ followed by $0.1 \mathrm{M}$ phosphate buffer containing $4 \%$ paraformaldehyde $(\mathrm{pH}=6.5$; $5 \mathrm{ml}$ ) to fix the brain. The brain was removed and post-fixed in $0.1 \mathrm{M}$ phosphate buffer containing $4 \%$ paraformaldehyde for $24 \mathrm{~h}$ and cryoprotected for $48 \mathrm{~h}$ in $0.1 \mathrm{M}$ phosphate buffer containing 30\% sucrose. The brain was then frozen and $30 \mu \mathrm{m}$ coronal sections were cut through the forebrain. The sections were stained with FJ (AG325-30MG; EMD Millipore (Darmstadt, Germany), Canada) according to the manufacturer's recommendations. The slides were then cover-slipped with Fluorescencepreserving VECTASHIELD Mounting medium (Vector, Burlingame, CA, USA; $\mathrm{H}-1,000$ ) and observed under a Zeiss (Thornwood, NY, USA) fluorescence microscope equipped with a computer-assisted image analysis system. The picture shown is representative of the damage observed in the striatum.

\section{Mouse stroke model}

C57bl/6 mice were used for the study. Ethical approval for the study was obtained from the Institutional Animal Care Committee (Dalhousie University). Hypoxic-ischemic (HI) brain injury was produced using a method adapted from Levine. ${ }^{37}$ The mice were anesthetized using isoflurane ( $3 \%$ with medical oxygen at a flow rate of $3 \mathrm{l} / \mathrm{min}$ ). The ventral portion of the neck was shaved and cleaned with soluprep and betadiene (SoluMed Inc; Laval, QC, Canada and Purdue Frederick Inc; Pickering, ON, Canada, respectively). Anesthesia was maintained with $2 \%$ isoflurane with an oxygen flow rate of $1.5 \mathrm{l} / \mathrm{min}$. A $1 \mathrm{~cm}$ incision was made with a scalpel to expose the sternohyoid and sternomastoid muscles. The left carotid artery was isolated and removed from the vagus nerve by blunt dissection. The carotid was then permanently occluded using a high-temperature electrocautery pen (Bovie Instruments; St. Perersberg, FL, USA). Incomplete occlusions and/or animals that displayed blood loss were immediately killed and excluded from the study. Following a $2-3 \mathrm{~h}$ recovery period, the animals were placed in a low-oxygen chamber (8\% oxygen balanced with nitrogen, flow rate $4 \mathrm{l} /$ $\mathrm{min})$. Following $50 \mathrm{~min}$ in the low-oxygen chamber, the mice were removed and individually housed to recover. Following a $4 \mathrm{~h}$ recovery, the mice were selected either for TTC (2,3,5-triphenyltetrazolium chloride, St. Louis, MO, USA) or water-free chloroform extraction. In both cases, the mice were humanely killed by intraperitoneal injection of an overdose of sodium pentobarbital overdose $(100 \mathrm{mg} / \mathrm{kg})$ and perfused intracardially with PBS. The mice were then decapitated and their brains rapidly removed from the cranial vault. For $\Pi \mathrm{TC}$ analysis, the brains were then placed in brain blocks and stored at $-20^{\circ} \mathrm{C}$ for $5 \mathrm{~min}$. Next, the brains were sliced into $1 \mathrm{~mm}$ coronal sections and placed in a $2 \%$ TTC solution at $37^{\circ} \mathrm{C}$ for $15 \mathrm{~min}$. The brain slices were then transferred to $4 \%$ formaldehyde for $20 \mathrm{~min}$. The slices were finally stored in $1 \%$ formaldehyde in PBS until photographic analysis. The brains used for extraction were harvested in the same manner as those for TTC staining. Once perfused and removed from the skull, the hindbrain and rostral forebrain were discarded. The brain was then cut along medial longitudinal fissure, dividing the brain into the affected and unaffected hemispheres. The brain sections were then immediately placed into methanol until further processing. The brain extraction was performed using the same protocol described for mitochondrial isolation.

\section{ABBREVIATIONS}

C-subunit, C-subunit of the mitochondrial ATP synthase; CSA, Cyclosporine A; mPTP, Mitochondrial Permeability Transition Pore; polyP, inorganic Polyphosphate; $\mathrm{PHB}$, polyhydroxybutyrate; TCL, thin layer chromatography.

\section{ACKNOWLEDGEMENTS}

This work was funded by Heart and Stroke Foundation of Canada (grants G-130003008 to EVP and G-15-0008818 to GSR) and American Heart Association (grant 16GRNT27260229 to EP).

\section{COMPETING INTERESTS}

The authors declare no conflict of interest. 


\section{REFERENCES}

1 Bernardi P, Krauskopf A, Basso E, Petronilli V, Blachly-Dyson E, Di LF et al. The mitochondrial permeability transition from in vitro artifact to disease target. FEBS $J$ 2006; 273: 2077-2099.

2 Piot C, Croisille P, Staat P, Thibault H, Rioufol G, Mewton N et al. Effect of cyclosporine on reperfusion injury in acute myocardial infarction. $N$ Engl J Med 2008; 359: 473-481.

3 Bonora M, Wieckowsk MR, Chinopoulos C, Kepp O, Kroemer G, Galluzzi L et al. Molecular mechanisms of cell death: central implication of ATP synthase in mitochondrial permeability transition. Oncogene 2015; 34: 1608.

4 Giorgio V, von SS, Antoniel M, Fabbro A, Fogolari F, Forte M et al. Dimers of mitochondrial ATP synthase form the permeability transition pore. Proc Natl Acad Sci USA 2013; 110: 5887-5892.

5 Bonora M, Bononi A, De ME, Giorgi C, Lebiedzinska M, Marchi S et al. Role of the c subunit of the FO ATP synthase in mitochondrial permeability transition. Cell Cycle 2013; 12: 674-683.

6 Alavian KN, Beutner G, Lazrove E, Sacchetti S, Park HA, Licznerski P et al. An uncoupling channel within the c-subunit ring of the F1FO ATP synthase is the mitochondrial permeability transition pore. Proc Natl Acad Sci USA 2014; 111: 10580-10585.

7 Halestrap AP, Richardson AP. The mitochondrial permeability transition: a current perspective on its identity and role in ischaemia/reperfusion injury. $J \mathrm{Mol}$ Cell Cardiol 2015; 78: 129-141.

8 Bernardi P. The mitochondrial permeability transition pore: a mystery solved? Front Physiol 2013; 4: 95.

9 Jonas EA, Porter Jr GA, Beutner G, Mnatsakanyan N, Alavian KN. Cell death disguised: the mitochondrial permeability transition pore as the c-subunit of the FF ATP synthase. Pharmacol Res 2015; 99: 382-392.

10 Bonora M, Wieckowski MR, Chinopoulos C, Kepp O, Kroemer G, Galluzzi L et al. Molecular mechanisms of cell death: central implication of ATP synthase in mitochondrial permeability transition. Oncogene 2015; 34: 1475-1486.

11 Chinopoulos C, Szabadkai G. What makes you can also break you, part III: mitochondrial permeability transition pore formation by an uncoupling channel within the C-subunit ring of the F1FO ATP synthase? Front Oncol 2014; 4: 235.

12 Pavlov E, Zakharian E, Bladen C, Diao CT, Grimbly C, Reusch RN et al. A large, voltage-dependent channel, isolated from mitochondria by water-free chloroform extraction. Biophys J 2005; 88: 2614-2625.

13 Reusch RN, Sadoff HL. Putative structure and functions of a poly-beta-hydroxybutyrate/calcium polyphosphate channel in bacterial plasma membranes. Proc Natl Acad Sci USA 1988; 85: 4176-4180.

14 Seidlmayer LK, Juettner VV, Kettlewell S, Pavlov EV, Blatter LA, Dedkova EN. Distinct mPTP activation mechanisms in ischaemia-reperfusion: contributions of $\mathrm{Ca} 2$ + , ROS, pH, and inorganic polyphosphate. Cardiovasc Res 2015; 106: 237-248.

15 Seidlmayer LK, Blatter LA, Pavlov E, Dedkova EN. Inorganic polyphosphate-an unusual suspect of the mitochondrial permeability transition mystery. Channels (Austin) 2012; 6: 463-467.

16 Seidlmayer LK, Gomez-Garcia MR, Blatter LA, Pavlov E, Dedkova EN. Inorganic polyphosphate is a potent activator of the mitochondrial permeability transition pore in cardiac myocytes. J Gen Physiol 2012; 139: 321-331.

17 Abramov AY, Fraley C, Diao CT, Winkfein R, Colicos MA, Duchen MR et al. Targeted polyphosphatase expression alters mitochondrial metabolism and inhibits calcium-dependent cell death. Proc Natl Acad Sci USA 2007; 104: 18091-18096.

18 Bernardi P. Modulation of the mitochondrial cyclosporin A-sensitive permeability transition pore by the proton electrochemical gradient. Evidence that the pore can be opened by membrane depolarization. J Biol Chem 1992; 267: 8834-8839.

19 Elustondo PA, Negoda A, Kane CL, Kane DA, Pavlov EV. Spermine selectively inhibits high-conductance, but not low-conductance calcium-induced permeability transition pore. Biochim Biophys Acta 2015; 1847: 231-240.
20 Papageorgiou AC, Hermawan S, Singh CB, Jendrossek D. Structural basis of poly(3hydroxybutyrate) hydrolysis by PhaZ7 depolymerase from Paucimonas lemoignei. J Mol Biol 2008; 382: 1184-1194.

21 Aschar-Sobbi R, Abramov AY, Diao C, Kargacin ME, Kargacin GJ, French RJ et al. High sensitivity, quantitative measurements of polyphosphate using a new DAPIbased approach. J Fluoresc 2008; 18: 859-866.

22 Bernardi P, Rasola A, Forte M, Lippe G. The mitochondrial permeability transition pore: channel formation by F-ATP synthase, integration in signal transduction, and role in pathophysiology. Physiol Rev 2015; 95: 1111-1155.

23 McGeoch JE, Guidotti G. A $0.1-700 \mathrm{~Hz}$ current through a voltage-clamped pore: candidate protein for initiator of neural oscillations. Brain Res 1997; 766: 188-194.

24 Elustondo PA, Angelova PR, Kawalec M, Michalak M, Kurcok P, Abramov AY et al. Polyhydroxybutyrate targets mammalian mitochondria and increases permeability of plasmalemmal and mitochondrial membranes. PLoS One 2013; 8: e75812.

25 Choi DW. Calcium: still center-stage in hypoxic-ischemic neuronal death. Trends Neurosci 1995; 18: 58-60.

26 Lee J, Ding S, Walpole TB, Holding AN, Montgomery MG, Fearnley IM et al. Organization of subunits in the membrane domain of the bovine F-ATPase revealed by covalent cross-linking. J Biol Chem 2015; 290: 13308-13320.

27 Reusch RN, Huang R, Bramble LL. Poly-3-hydroxybutyrate/polyphosphate complexes form voltage-activated $\mathrm{Ca} 2+$ channels in the plasma membranes of Escherichia coli. Biophys J 1995; 69: 754-766.

28 Das S, Lengweiler UD, Seebach D, Reusch RN. Proof for a nonproteinaceous calcium-selective channel in Escherichia coli by total synthesis from (R)-3-hydroxybutanoic acid and inorganic polyphosphate. Proc Natl Acad Sci USA 1997; 94: 9075-9079.

29 Das S, Reusch RN. Gating kinetics of E. coli poly-3-hydroxybutyrate/polyphosphate channels in planar bilayer membranes. J Membr Biol 1999; 170: 135-145.

30 Seebach D, Fritz MG. Detection, synthesis, structure, and function of oligo(3hydroxyalkanoates): contributions by synthetic organic chemists. Int J Biol Macromol 1999; 25: 217-236.

31 Chalmers S, Nicholls DG. The relationship between free and total calcium concentrations in the matrix of liver and brain mitochondria. J Biol Chem 2003; 278 : 19062-19070.

32 Solesio ME, Demirkhanyan L, Zakharian E, Pavlov EV. Contribution of inorganic polyphosphate towards regulation of mitochondrial free calcium. Biochim Biophys Acta 2016; 1860: 1317-1325.

33 Solesio ME, Elustondo PA, Zakharian E, Pavlov EV. Inorganic polyphosphate (polyP) as an activator and structural component of the mitochondrial permeability transition pore. Biochem Soc Trans 2016; 44: 7-12.

34 Kinnally KW, Campo ML, Tedeschi H. Mitochondrial channel activity studied by patch-clamping mitoplasts. J Bioenerg Biomembr 1989; 21: 497-506.

35 Szabo I, Zoratti M. The giant channel of the inner mitochondrial membrane is inhibited by cyclosporin A. J Biol Chem 1991; 266: 3376-3379.

36 Cao C, Yudin Y, Bikard Y, Chen W, Liu T, Li H et al. Polyester modification of the mammalian TRPM8 channel protein: implications for structure and function. Cell Rep 2013; 4: 302-315.

37 Levine S. Anoxic-ischemic encephalopathy in rats. Am J Pathol 1960; 36: 1-17.

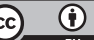

This work is licensed under a Creative Commons Attribution 4.0 International License. The images or other third party material in this article are included in the article's Creative Commons license, unless indicated otherwise in the credit line; if the material is not included under the Creative Commons license, users will need to obtain permission from the license holder to reproduce the material. To view a copy of this license, visit http://creativecommons.org/licenses/ by/4.0/

(c) The Author(s) 2016 